\title{
How respiratory system mechanics may help in minimising ventilator-induced lung injury in ARDS patients
}

\author{
P.P. Terragni, G.L. Rosboch, A. Lisi, A.G. Viale, V.M. Ranieri
}

How respiratory system mechanics may help in minimising ventilator-induced lung injury in ARDS patients. P.P. Terragni, G.L. Rosboch, A. Lisi, A.G. Viale, V.M. Ranieri. (C) ERS Journals Ltd 2003.

ABSTRACT: The main supportive therapy in acute respiratory distress syndrome patients is mechanical ventilation. As with any therapy, mechanical ventilation has sideeffects, and may induce lung injury (ventilator-induced lung injury (VILI)/ventilatorassociated lung injury). The mechanical factors responsible for VILI are thought to be related to tidal recruitment/derecruitment of previously collapsed alveoli and/or pulmonary overdistension.

The volume/pressure $(V I P)$ curve of the respiratory system in patients as well as in animal models of acute lung injury (ALI) has a characteristic sigmoid shape, with a lower inflection point (LIP) corresponding to the pressure/end-expiratory volume required to initiate recruitment of collapsed alveoli, and an upper inflection point (UIP) corresponding to the pressure/end inspiratory volume at which alveolar overdistension occurs. "Protective" ventilatory approaches have therefore set out to minimise mechanical injury by using the VIP curve to individualise positive end-expiratory pressure (PEEP) (PEEP above the LIP) and tidal volume (by setting end-inspiratory $V I P$ below the UIP) since a large number of experimental studies correlate $P I V$ curves to histological and biological manifestations of VILI and two randomised trials showed that protective ventilatory strategy individually tailored to the $P I V$ curve minimised pulmonary and systemic inflammation and decreased mortality in patients with ALI.

However, despite the fact that several studies have: 1) proposed new techniques to perform pressure/volume curves at the bedside, 2) confirmed that the lower inflection point and upper inflection point correspond to computed tomography scan evidence of atelectasis and overdistension, and 3) demonstrated the ability of the pressure/volume curve to estimate alveolar recruitment with positive end-expiratory pressure, no large studies have assessed whether such measurement can be performed in all intensive care units as a monitoring tool to orient ventilator therapy. Preliminary experimental and clinical studies show that the shape of the dynamic inspiratory pressure/time profile during constant flow inflation (stress index), allows prediction of a ventilatory strategy that minimises the occurrence of ventilator-induced lung injury. Eur Respir J 2003; 22: Suppl. 42, 15s-21s.
Università di Torino, Dipartimento di discipline Medico-Chirurgiche, Sezione di Anestesiologia e Rianimazione, Ospedale S. Giovanni Battista, Torino, Italy.

Correspondence: V.M. Ranieri

Università di Torino

Dipartimento di discipline Medico-Chirurgiche Sezione di Anestesiologia e Rianimazione

Ospedale S. Giovanni Battista

Corso Dogliotti 14

10126 Torino

Italy

Fax: 390116960448

E-mail: marco.ranieri@unito.it

Keywords: Acute respiratory distress syndrome mechanical stress

mechanical ventilation

respiratory mechanics

stress index

ventilator-induced lung injury

Supported by Cofinanziamento 2002-2004 Ministero dell' Istruzione, dell'Università e della Ricerca.
The acute respiratory distress syndrome (ARDS) is a common disease with devastating clinical effects. Mechanical ventilation is the main supportive therapy to re-establish sufficient oxygen supply to peripheral organs. As with any therapy, mechanical ventilation may expose patients to sideeffects. Clinicians quickly recognised that mechanical ventilation could cause alveolar rupture and air leak, the so-called barotrauma [1]. In 1974, WEBB and TIERNEY [2] showed that mechanical ventilation could also be responsible of ultrastructural injury, independently of air leaks. The potential clinical implication of these data was not realised until a series of studies showed that, apart from the physical alveolar disruption, mechanical ventilation can induce further injury to the lung by increasing alveolar-capillary permeability through the overdistension of the lung (volutrauma) [3] and/ or worsening lung injury through the tidal recruitmentderecruitment of the collapsed alveoli (atelectrauma) [4, 5], and lead to even more subtle injury manifested by the activation of the inflammatory process (biotrauma) [6-8]. All these experimental and clinical data led to the concept that all the pathophysiological mechanisms involved in ARDS (ventilation-perfusion mismatch and reduced compliance, lung oedema, atelectasis, pulmonary inflammation) may be worsened by the mechanical stress caused by inappropriate ventilator settings. In the early 1990s, an international consensus conference concluded that both tidal overdistension of normal alveoli and opening-closing of collapsed alveoli, contribute to a component of a progressive lung injury that arises not only from the disease process itself, but also from the impact of the ventilator patterns applied during the course of the disease [9]. Ventilator-induced lung injury (VILI) was therefore defined as acute lung injury directly induced by mechanical ventilation in animal models whereas ventilatorassociated lung injury (VALI) was defined as lung injury that resembles ARDS and that occurs in patients receiving mechanical ventilation $[10,11]$.

Although randomised clinical trials $[8,12,13]$ have successfully demonstrated that ventilatory strategy designed to minimise overdistension and opening-closing may reduce mortality in patients with ARDS, information regarding the 
bio-mechanical characteristics of stress applied to the ventilated lungs are still missing. VALI is in fact determined by the dynamic and continuous interaction between 1) the mechanical characteristics of the lung and 2) the ventilator settings. The relationship between these terms is conditioned by the dynamic variations in respiratory mechanics as determined by the status and evolution of the pathological process and by the consequences of ventilator parameters on the mechanical characteristics of the lung. Therefore clinicians have to choose tidal volume, positive end-expiratory pressure (PEEP) and recruiting manoeuvres assuming that the ventilator settings are not causing VALI but lacking a clinical tool able to identify whether or not the interaction between the currently used ventilator settings and the actual status of pulmonary mechanics is resulting in mechanical stress.

The mechanical characteristics of animal models $[4,6,14]$ and patients $[15,16]$ with ARDS have been investigated by the analysis of the static pressure/volume $(P / V)$ curve of the respiratory system. Besides this, analysis of the $P / V$ curve provided most of the physiological rationale explaining the pulmonary injury due to VILI/VALI. The static $P / V$ curve is in fact characterised by a lower (LIP) and an upper (UIP) inflection point that are thought to represent the average critical opening pressure above which alveolar units start to re-open and the $V / P$ values above which stretching and overdistension start to occur, respectively [17]. Several studies demonstrated that tidal inflation starting below the LIP on the $P / V$ curve leads to tidal recruitment/derecruitment of previously collapsed alveoli while tidal ventilation occurring above the UIP results in pulmonary overstretching both leading to a spectrum of pulmonary and systemic lesions such as air leaks [18], alterations in lung fluid balance [3], increases in endothelial and epithelial permeability $[19,20]$, severe tissue damage [4], and pulmonary [6] and systemic [11, 21] production of inflammatory mediators.

Because of this link between VILI/VALI and assessment of the $P / V$ curve, and in an effort to make the measurement of the $P / V$ curve available at the bedside, a growing interest in the development of new technologies, and on the clinical interpretation of the $P / V$ curve has become evident in the last few years [22-30]. However, although a large number of experimental studies correlated $P / V$ curves to histological [4] and biological [6, 21] manifestations of VILI, only two randomised trials showed that protective ventilatory strategy individually tailored to the $P / V$ curve minimised pulmonary and systemic inflammation [8] and decreased mortality in patients with ALI [12]. Furthermore, despite the fact that several studies have proposed new techniques to perform $P / V$ curves at the bedside $[23,24,27]$, confirming that the LIP and UIP correspond to computed tomography (CT) scan evidence of atelectasis and overdistension [26, 28, 29] and demonstrating the ability of the $P / V$ curve to estimate alveolar recruitment with PEEP $[15,16]$, no large clinical studies have assessed whether such measurement can be performed in all intensive care units as a monitoring tool to orient ventilator therapy.

This chapter will: 1) review the basic principles of mechanical stress, 2) discuss how to measure and interpret the static $P / V$ curve to minimise VILI/VALI, 3) revise the potential advantage of the use of the dynamic $P / V$ curve to monitor, prevent and minimise VILI/VALI.

\section{Biophysical characteristics of the lungs relative to mechanical stress}

MEAD et al. [31] examined the intrapulmonary distribution of pressure in a lung model that included normal and collapsed alveoli. In such a model tidal inflation meant that: 1) part of the alveoli continuously accessible to ventilation was overinflated; and 2) part of the collapsed alveoli were continuously recruited and derecruited. Under these circumstances when tidal inflation generated a pressure of $30 \mathrm{cmH}_{2} \mathrm{O}$ at the airway opening, in the area encompassed between the hyperinflated and the normal alveoli and between the continuously recruited-derecruited alveoli and the normally expanded regions, the interstitial pressure was amplified up to $140 \mathrm{cmH}_{2} \mathrm{O}$. This distribution of pressure may cause the generation of two sources of mechanical distortion [31-35]: shear stress and stress failure.

\section{Shear stress}

Shear stress is a form of mechanical stress generated when blood or air move across a cell surface, thereby generating a force parallel to the plasma membrane that induces a tangential distortion of the cell. In damaged lungs the development of shear stress is related to the cyclic opening and closing of small airways induced by recruitment/derecruitment of alveolar units. Diseased lungs with a heterogeneous distribution of lesions may be subjected to a much greater regional stress than homogeneous lungs $[4,31]$. The occlusion of small airways by exudate or apposition of their walls requires high airway pressure to restore patency, resulting in shear stress and damage of the airways, particularly if the cycle is continuously repeated [36]. Airway collapse and the consequent recruitment-derecruitment may not occur in normal lungs, being favoured by surfactant deficiency and lung disease, modifying the interstitial support of the airways [35].

\section{Stress failure}

Stress failure depends on the development of excessive wall stress, defined as the ratio of alveolar wall tension to thickness. The limited strength of the alveolar-capillary barrier may explain such a mechanism of mechanical stress. It has been known that high airway pressure between the alveolus and the vascular bed during positive pressure ventilation causes the passage of air across the epithelial surface, along the bronchovascular sheath and then into the interstitial tissues $[32,33]$. The endothelium, so close to the epithelial surface, is subject to stress failure determined by forces derived both from transpulmonary and intravascular pressures [32]. Fu et al. [33] showed that at a constant transmural pressure, an increase of transpulmonary pressure from 5 to $20 \mathrm{cmH}_{2} 0$ produced a significant increase in the number of epithelial and endothelial breaks. There was a further increase in number of breaks at the same transpulmonary pressure when capillary transmural pressure was increased. The local or regional stress induced by lung inflation may increase microvascular transmural pressures with disruption of capillaries ("capillary stress failure"), determining changes in the alveolo-capillary barrier [34]. The forces generated by mechanical ventilation may therefore interact with those due to pulmonary vascular perfusion to increase lung injury.

The mechanical characteristics of the lungs are conditioned by the mechanics of its microstructure and the relative content of elastin and collagen in the alveolar walls. The alveolar septum is composed of a skeleton of collagen and elastin fibres that are interlaced with the capillary network. Many models of lung parenchyma have used various geometrical bodies to describe the volumetric structure of the alveolar region. To investigate fibre organisation and the potential distribution of mechanical deformation and stress, a twodimensional model of the alveolar sac was proposed [37]. 
Two-dimension electron microscope scanning showed that the traction stresses $(\sigma)$ at different lung volumes were detected with an analysis of internal stresses within the septal walls. Characteristics of lung tissue were evaluated through measurements of length-tension [37, 38]. The authors found the following relationship:

$$
\sigma=\lambda^{\mathrm{n} 1}-\lambda^{-\mathrm{n} 2}
$$

where $\sigma$ represents the stress, $\lambda$ the stretch ratio. This equation therefore identifies different traction stresses $(\sigma)$ at different lung volumes and alveolar pressures. Experimental studies showed that at $60 \%$ total lung capacity, tensions are up to 6 times higher than those at resting lung volume. In contrast, the tension stress within internal walls shared by adjacent alveoli, is nearly zero thus confirming that alveolar distortion is not uniform.

\section{The use of the static pressure/volume curve to minimise ventilator-induced lung injury/ventilator-associated lung injury}

Patients with ARDS are characterised by a reduction of the range of volume excursion, because of the reduction in the ventilating units, and a smaller change in volume for unit of change in pressure. The initial part of the $P / V$ curve, at very low lung volume, is therefore considerably flatter than the rest of the curve, showing the amount of pressure required to open collapsed peripheral alveoli. This "lower inflection point", separates a tract of the curve with bad elastic properties from the tract characterised by optimal elastic properties. After this initial tract, the curve presents a linear section in which the open alveoli are ventilated. Then the VIP curve flattens again at values of tidal volume lower than those observed in normal subjects. This "upper inflection point" indicates that stretching and overdistension of at least some alveolar structures is occurring.

\section{Lower inflection point}

Inflation of an excised lung requires a critical opening pressure to be applied in order to re-expand the collapsed alveoli [39]. This critical pressure appears on the $P / V$ curve as the pressure corresponding to the sudden change in slope of the curve after the initial inflation. In normal subjects, this critical opening pressure amounts to $\sim 20 \mathrm{cmH}_{2} \mathrm{O}$. Similarly, in patients with ARDS, the inspiratory $P / V$ curve shows a LIP, that is the sudden change in slope occurring at the onset of tidal inflation, when the applied pressure varies between $10-20 \mathrm{cmH}_{2} \mathrm{O}$. This shows that in ARDS the vast majority of the lung is collapsed at the beginning of inspiration. The pressure corresponding to the LIP should therefore represent the minimal level of PEEP that should be applied in order to have tidal inflation within an open lung. Considering that ARDS and ALI are conditions of not homogeneous lung parenchyma, densities, evaluated with CT are more concentrated in dependent lung regions, where there is a more positive pleural pressure if compared with nondependent regions $\left(0 \mathrm{cmH}_{2} \mathrm{O}\right.$ and $-3,-5 \mathrm{cmH}_{2} \mathrm{O}$ respectively). The influence of this vertical gradient in pleural pressure in the supine position may be enhanced by the gravitational distribution of oedema.

\section{Upper inflection point}

The decrease of $P / V$ curve slope indicates the end of alveolar recruitment, the beginning of alveolar overdistension and so the maximal alveolar pressure that should be applied to obtain the maximal amount of alveolar recruitment. In normal subjects, the UIP is reached at a lung volume that is $85-90 \%$ of total lung capacity (TLC); in patients with ARDS UIP occurs at a much lower volume. An increase in pressure above the UIP only gives overdistension without any other increase in volume, with a maximal stretch of lung aerated areas.

\section{Technique to assess the static pressurelvolume curve}

The super-syringe technique. The super-syringe technique was the first technique used to assess the status of elastic properties of the respiratory system in mechanically ventilated ALI/ ARDS patients in supine position sedated and paralysed, to permit the slow inflation of the lung with predetermined gas volume of oxygen. The inflated volume is $100-200 \mathrm{~mL}$. The syringe stops for $2-3 \mathrm{~s}$, then the respiratory system is inflated with intermittent pause until a volume of $25 \mathrm{~mL} \cdot \mathrm{kg}^{-1}$ or an airway pressure of $40 \mathrm{cmH}_{2} \mathrm{O}$ are reached. With this technique it is easy to detect the LIP and UIP but, on the other hand, paralysis, sedation and disconnection of the patient from the ventilator are required.

Rapid airway occlusion technique. The rapid airway occlusion technique is based on a single-breath occlusion at different inflation during mechanical ventilation. With inspiratory constant flow different volumes are achieved. Each occlusion is maintained until a plateau in the open airways pressure is obtained thus representing the static pressure of the total respiratory system. Using different volumes the static $V I P$ curve can be constructed. Advantages of this technique include no need for patient disconnection and the ability to identify the elastic properties of the respiratory system as determined by the actual volume. Besides, the measurement does not require special devices. However, patients must be paralysed and sedated and curves are not immediately available since single data points need to be first collected and recorded and then plotted; identification of LIP and UIP is not easy.

Constant flow technique. The constant flow technique is based on the assumption that when inspiratory flow is constant during passive inflation the rate of change in the airway opening pressure is related to the elastance of the respiratory system and the resistive components are nil. There is no need to disconnect the patient from the ventilator, special devices are not required and results are available at the bedside; LIP and UIP are usually easily identified. Yet, this method requires paralysis, sedation and only a few ventilators are equipped with such a monitoring tool.

In patients with ARDS, the rapid airway occlusion technique (static VIP curve) provides the same information as the constant flow technique (with a flow of $3 \mathrm{~L} \cdot \mathrm{min}^{-1}$ ) regarding the elastic properties of the respiratory system, whereas the $V I$ $P$ curve obtained by the $9 \mathrm{~L} \cdot \mathrm{min}^{-1}$ constant flow is slightly shifted to the right [27]. The slopes of the VIP curves and the LIP are not different between all methods, indicating that the resistive component induced by administering a constant flow $\leqslant 9 \mathrm{~L} \cdot \mathrm{min}^{-1}$ is not of clinical relevance. However all methods have an intrinsic risk of adverse effects, including hypoxaemia at low lung volumes and derecruitment at low levels of PEEP [26-28]. Other problems include haemodynamic changes (decrease of venous return) or complications related to sedation or paralysis required to obtain the characteristics of passive mechanics of the respiratory system. For all these reasons, $P / V$ curves are not usually obtained in the routine clinical assessment [39].

A nonlinear model of respiratory mechanics in ARDS has recently been used to verify the physiological interpretation of 
the LIP and UIP and to examine their potential use in the clinical setting to set mechanical ventilation [30, 40]. This analysis showed that: 1) the initial increase in slope of the $V I P$ curve indicates the minimal pressure at which alveolar recruitment starts to occur rather than the maximum level of PEEP able to provide maximum recruitment. Under these circumstances, a PEEP level equal to the LIP underestimates the optimal level of PEEP able to minimise end-expiratory alveolar collapse; 2) the decrease in the slope of the $V / P$ curve does not indicate the beginning of alveolar overdistension but the end of alveolar recruitment. According to this mathematical model, the UIP is therefore unrelated to alveolar overdistension being caused by the decrease in rate of alveolar recruitment during lung inflation. Under these circumstances, the LIP will indicate the maximal alveolar pressure that should be applied to obtain the maximal amount of alveolar recruitment. Animal and clinical experiments are consistent with these data and have shown that recruitment occurs throughout the entire lung inflation from end-expiratory lung volume to TLC rather than being an "all or none" phenomenon [41, 42].

\section{Analysis of the dynamic pressure/time curve during constant flow: a "stress index" to minimise ventilator- induced lung injury/ventilator-associated lung injury}

The current authors recently proposed that the use of the static $P / V$ curve could be replaced by the analysis of the dynamic airway opening pressure/time $(P / \mathrm{t})$ profile during constant-flow inflation [43]. In 19 patients with ALI the present authors previously found that a downward concavity on the $P /$ t profile during constant flow inflation corresponded to a static $P / V$ curve with a distinct LIP and a continuous increase in compliance (i.e. progressive recruitment with inflating volume) [15]. On the other hand, an upward concavity on the $P / \mathrm{t}$ profile during constant flow inflation corresponded to a static $P / V$ curve with a distinct UIP and a continuous reduction in compliance (i.e. progressive overdistension with inflating volume) [15]. Based on these results the current authors raised the hypothesis that analysis of the shape of the $P / t$ curve during constant flow inflation could identify the presence of tidal recruitment and/or tidal overinflation and therefore allow a noninvasive and continuous assessment of mechanical stress due to nonappropriate ventilator settings.

During constant flow conditions and if resistances are constant, airway opening pressure $(P$ ao $)$ changes linearly with time when compliance does not change with increasing lung volume. When compliance decreases, $P$ ao is concave upward and when compliance increases $P$ ao is concave downward with respect to the time axis $[15,4446]$. Such an analysis of the $P / \mathrm{t}$ relationship is based on the assumption that during volume controlled ventilation with a constant flow inflation, the rate of change of pressure is related to the changes in pulmonary compliance $[15,4446]$. Under these circumstances, the $P$ ao profile as function of inspiratory time $(\mathrm{t})$ can be described by a power equation (fig. 1):

$$
P \text { ao }=a \cdot t^{b}+c
$$

The coefficient $a$ is a scaling factor, $c$ is the pressure value at $\mathrm{t}=0$. The coefficient $b$ is a dimensionless number that describes the shape of the $P /$ t curve and that can therefore identify and quantify mechanical stress (stress index): a stress index $=1$ means that the $P / t$ curve is linear and compliance remains constant throughout tidal inflation; a stress index $<1$ indicates that the $P /$ t curve has a downward concavity due to the tidal increase in compliance therefore identifying stress due to tidal recruitment; a stress index $>1$ indicates that the $P / t$ curve has an upward concavity due to the tidal reduction in compliance therefore identifying stress due to tidal overinflation. This analysis requires several assumptions: 1) a stiff chest wall may influence estimation of the upward/downward concavity on the dynamic $P /$ curve [44]. 2) In more complex conditions, the $P / t$ curve may be characterised by a sigmoidal shape with an initial downward concavity due to alveolar opening, followed by a linear portion and a final downward concavity due to alveolar overdistension. Under these circumstances, it would be best to fit the power equation first to the initial portion of the curve (to set PEEP) and then to the second portion of the curve (to set tidal volume $(V \mathrm{~T})$ ). 3) On a theoretical basis, the time course of applied pressure during constant flow inflation should be characterised by an immediate step change due to the resistive components, abruptly followed by the progressive increase in pressure reflecting the changes in pulmonary compliance [45]. However, on- and off flow transients may be due to pendelluft (i.e. the time required to achieve a steadystate flow to each alveolar unit with different time constants) [47], viscoelasticity [30,48] and the time required by the ventilator to initiate and to stop delivery of constant flow [15]. The first part of the pressure events must therefore be discarded, and only the portion on the $P /$ t relationship corresponding to constant flow remains valid. 4) A high sampling frequency of the recorded signals is required to achieve an adequate dynamic recording of airway pressure with no phase lag at high frequency. 5) Resistive and viscoelastic contribution to airway pressure are assumed constant over the range of changes in lung volume. Some of these factors may explain the relative low specificity (i.e. a relevant number of false positive) of the dynamic $P / t$ profile to detect VILI.

The "stress index" approach was initially tested in an isolated lavage model of ARDS [43]. A significant $(p<0.0001)$ U-shaped relationship between individual values of stress index and pulmonary histological damage and pulmonary concentration of pro-inflammatory cytokines was found; the lowest values of histological injury score and cytokine concentration was systematically associated with a stress index $\sim 1$. The threshold value for the stress index that discriminated best between lungs with and without histological and inflammatory evidences of VILI ranged between $0.90-1.10$. For such threshold values, the sensitivity of the stress index to identify noninjurious ventilatory strategy was 1.00 . A second study [49] examined the impact of different ventilatory strategies on the development of ischemia-reperfusion injury following lung transplantation and evaluated whether a ventilatory strategy aimed to maintain a stress index of 1 would minimise pathophysiological indices of ARDS in the setting of lung transplantation. In a rat lung transplant model, animals were randomised into two groups defined by the ventilatory strategy during the early reperfusion period.

In the conventional mechanical ventilation group the transplanted lung was ventilated with a $V T$ equal to $50 \%$ of the inspiratory capacity of the left lung and a low PEEP. In the minimal mechanical stress ventilation group the transplanted lung was ventilated with a $V$ T equal to $20 \%$ of the inspiratory capacity of the left lung and PEEP adjusted according to the shape of the $P / t$ curve to obtain a stress index of 1 . After $3 \mathrm{~h}$ of reperfusion, oxygenation from the transplanted lung was significantly higher in animals ventilated with a stress index of 1 than in animals ventilated with conventional ventilation. In addition, elastance, cytokine levels, and morphological signs of injury were significantly lower in the group ventilated with a stress index of 1 . This study demonstrates that the mode of mechanical ventilation used in the early phase of reperfusion of the transplanted lung can influence ischemia-reperfusion injury, and a protective ventilatory strategy based on a stress index of 1 can lead to improved lung function after lung transplantation. In a rabbit 

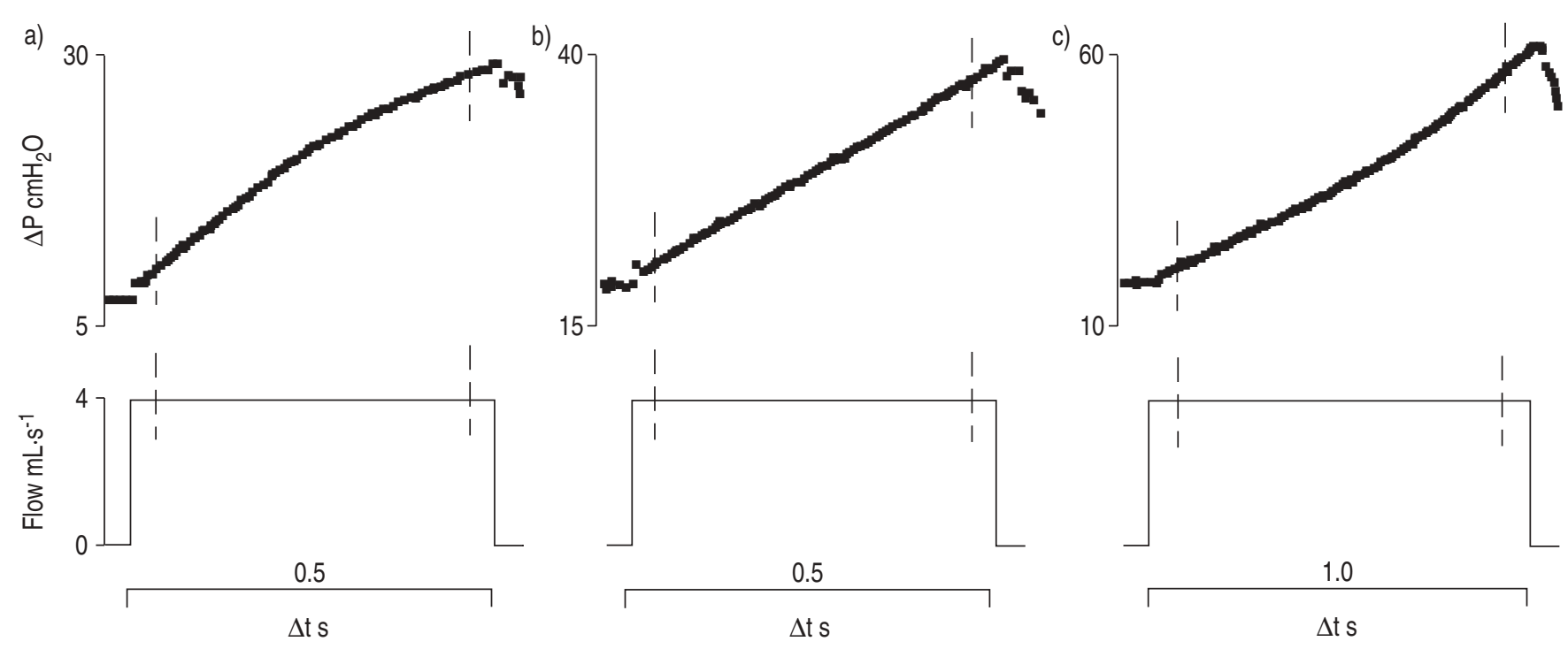

Fig. 1. - The conceptual illustration of the dynamic pressure/time $(P / \mathrm{t})$ curve used in the current study. Based on the power equation $P \mathrm{~L}=a \cdot \mathrm{t}^{b}+c$ (where $P_{\mathrm{L}}=$ transpulmonary pressure; $a=$ slope of the $P / \mathrm{t}$ relationship at time $=1 \mathrm{~s} ; b=$ dimensionless number that describes the shape of the $P / \mathrm{t}$ curve; c=pressure at time $=0$ ); a) $b=0.7$ (a stress index $<1$ ) produces a convex $P /$ curve, indicating continuing recruitment; b) $b=1.01(\mathrm{a}$ stress index $=1$ ) produces a straight $P / t$ line, indicating no alveolar continuing recruitment or overdistension; and c) $b=1.32$ (a stress index $>1)$ produces a concave $P / \mathrm{t}$ curve, indicating alveolar overdistension. The power equation was applied to the $P \mathrm{~L}$ signal during a constant inspiratory flow (vertical bars) [43].

ARDS model, NAKANE et al. [50] compared efficacy to minimise VILI of a ventilatory strategy aimed to maintain a stress index of 1 to the National Institutes of Health (NIH) protective ventilatory strategy [13]. Animals were randomly ventilated for $3 \mathrm{~h}$ using one of the following ventilatory strategies: stress index group: $V \mathrm{~T} 6 \mathrm{~mL} \cdot \mathrm{kg}^{-1}$, PEEP adjusted so that $\mathrm{b}=1$; NIH group: $V \mathrm{~T} 6 \mathrm{~mL} \cdot \mathrm{kg}^{-1}$, PEEP set according to the table created by NIH ARDSNet; Injurious group: $V \mathrm{~T}$ 10-12 $\mathrm{mL} \cdot \mathrm{kg}^{-1}$, PEEP $1-2$, inspiratory oxygen fraction was adjusted so that arterial oxygen tension was 55-80 in all groups. Respiratory mechanics worsened with time only in animals ventilated with the NIH and the injurious ventilatory strategies while they were maintained at the baseline levels only in animals ventilated with a stress index of 1 . After $3 \mathrm{~h}$ of mechanical ventilation, lung homogenate concentration of interleukin- 8 in the dependent region was significantly lower in animals protected by VILI using the stress index strategy than in animals protected using the NIH strategy; histological exams showed significantly lower incidence of VILI in the stress index group compared to the other groups. In eight pigs lung injury was induced with lung lavage [51]. Each animal was ventilated in random order with three ventilator settings aimed to obtain a stress index $=1$ a stress index $>1$ and a stress index $<1$. At the end of each experimental condition the current authors measured respiratory mechanics, gas exchange and quantified tidal recruitment and tidal overinflation with spiral CT scan and multiple inert gas elimination technique (MIGET). As can be seen in table 1, CT evidences of intratidal alveolar collapse and/or overdistension are mirrored by the stress index. Preliminary data in patients with ARDS $[52,53]$ show that the use of the stress index is feasible also in the clinical scenario and that, compared to the gold standard protective ventilatory strategy (NIH protocol), patients ventilated with a stress index of 1 have lower elastance and higher recruitment of collapsed alveoli.

Other groups have independently confirmed and expanded these findings. In 20 paralysed children with acute respiratory distress syndrome, NEVE et al. [54] showed that the analysis of the profile of the dynamic pressure/time curve during constant flow ventilation permits detection of hyperinflation and has a good agreement with the results of the static pressure/volume curve. GAMA DE ABREU et al. [55] in a rabbit model of unilateral acute respiratory distress syndrome confirmed that the analysis of the pressure/time curve during constant flow ventilation allows detection of optimal protective strategy also during one-lung ventilation. These data therefore suggest that the shape of the dynamic inspiratory pressure/time profile during constant flow inflation (stress index) allows prediction of a ventilatory strategy that minimises the occurrence of ventilator-induced lung injury. Modern ventilators are able to

Table 1.- Values of tidal recruitment and hyperinflation (computed tomography scan technique) and shunt and dead space (multiple inert gas elimination technique (MIGET)) in eight pigs with acute respiratory distress syndrome (ARDS) and ventilated with a stress index $=1,>1$ and $<1[51]$

\begin{tabular}{lccc}
\hline & Stress index $<1$ & Stress index=1 & Stress index $>1$ \\
\hline Coefficient $b$ & $0.78 \pm 0.1$ & $1.09 \pm 0.05^{* * *}$ & $1.37 \pm 0.07^{* * *}$ \\
$V \mathrm{~T} \mathrm{~mL} \cdot \mathrm{kg}^{-1}$ & $12.7 \pm 2$ & $6.9 \pm 0.8^{* * *}$ & $12.7 \pm 2^{* * *}$ \\
PEEPtot cm ${ }_{2} \mathrm{O}$ & $1.4 \pm 1$ & $15.4 \pm 3.5^{* * *}$ & $14.6 \pm 1.6^{* * *}$ \\
Tidal recruitment g & $14.1 \pm 6$ & $0.8 \pm 1^{* * *}$ & $1 \pm 1.4^{* * *}$ \\
Tidal hyperinflation g & $1 \pm 0.2$ & $1.3 \pm 0.3$ & $4.5 \pm 0.5^{* * *}$ \\
Shunt \% Qt & $30 \pm 10$ & $3 \pm 3^{* * *}$ & $1 \pm 1^{* * *}$ \\
Alveolar dead space $\mathrm{mL}$ & $60 \pm 10$ & $50 \pm 10$ & $125 \pm 11^{* * *}$ \\
\hline
\end{tabular}

Qt: cardiac output; VT: tidal volume; PEEPtot: total positive end-expiratory pressure. ${ }^{* *}: \mathrm{p}<0.001$. 
deliver excellent square-wave inspiratory flow profiles, and are also equipped with monitoring tools that are able to provide on-line, dynamic pressure/time curves, however further clinical studies will be required to confirm the utility of this approach to set protective ventilatory strategies and minimise ventilator-induced lung injury.

\section{References}

1. Macklin MT, Macklin CC. Malignant interstitial emphysema of the lungs and mediastinum as an important occult complication in many respiratory disease and other conditions: an interpretation of the clinical literature in the light of laboratory experiment. Medicine 1944; 23: 281-352.

2. Webb HH, Tierney DF. Experimental pulmonary edema due to intermittent positive pressure ventilation with high inflation pressures. Protection by positive end-expiratory pressure. Am Rev Respir Dis 1974; 110: 556-565.

3. Dreyfuss D, Soler P, Basset G, Saumon G. High inflation pressure pulmonary edema. Respective effects of high airway pressure, high tidal volume, and positive end-expiratory pressure. Am Rev Respir Dis 1988; 137: 1159-1164.

4. Muscedere JG, Mullen JB, Gan K, Slutsky AS. Tidal ventilation at low airway pressures can augment lung injury. Am J Respir Crit Care Med 1994; 149: 1327-1334.

5. Slutsky AS. Lung injury caused by mechanical ventilation. Chest 1999; 116: 9S-15S.

6. Tremblay L, Valenza F, Ribeiro SP, Li J, Slutsky AS. Injurious ventilatory strategies increase cytokines and c-fos m-RNA expression in an isolated rat lung model. $J$ Clin Invest 1997; 99: 944-952.

7. Tremblay LN, Slutsky AS. Ventilator-induced injury: from barotrauma to biotrauma. Proc Ass Am Physicians 1998; 110: 482-488.

8. Ranieri VM, Suter PM, Tortorella C, et al. Effect of mechanical ventilation on inflammatory mediators in patients with acute respiratory distress syndrome: a randomized controlled trial. JAMA 1999; 282: 54-61.

9. International Consensus Conference ATS. Ventilator-associated lung injury in ARDS. Am J Respir Crit Care Med 1999; 160: 2118-2124.

10. Slutsky AS. Mechanical ventilation. Chest 1993; 10: 18331859.

11. Slutsky AS, Tremblay LN. Multiple system organ failure. Is mechanical ventilation a contributing factor? Am J Respir Crit Care Med 1998; 157: 1721-1725.

12. Amato MB, Barbas CS, Medeiros DM, et al. Effect of a protective-ventilation strategy on mortality in the acute respiratory distress syndrome. N Engl J Med 1998; 338: $347-$ 354.

13. Acute Respiratory Distress Syndrome Network. Ventilation with lower tidal volumes as compared with traditional tidal volumes for acute lung injury and the acute respiratory distress syndrome. The Acute Respiratory Distress Syndrome Network. N Engl J Med 2000; 342: 1301-1308.

14. Martin-Lefreve L, Roupie E, Dreyfuss D, Saumon G. Can respiratory system pressure volume (PV) curve analysis predict the occurrence of volutrauma? Am J Respir Crit Care Med 1998; 157: A693.

15. Ranieri VM, Giuliani R, Fiore T, Dambrosio M, Milic-Emili J. Volume-pressure curve of the respiratory system predicts effects of PEEP in ARDS: "occlusion" versus "constant flow" technique. Am J Respir Crit Care Med 1994; 149: 19-27.

16. Ranieri VM, Eissa NT, Corbeil C, et al. Effects of positive end-expiratory pressure on alveolar recruitment and gas exchange in patients with the adult respiratory distress syndrome. Am Rev Respir Dis 1991; 144: 544-551.

17. Brochard L. Respiratory pressure-volume curves. In: Tobin MJ (ed). Principles and practice of intensive care monitoring. New York, McGraw-Hill, 1997; pp. 597-616.
18. Tobin MJ. Advances in mechanical ventilation. $N$ Engl J Med 2001; 344: 1986-1996.

19. Dreyfuss D, Basset G, Soler P, Saumon G. Intermittent positive-pressure hyperventilation with high inflation pressures produces pulmonary microvascular injury in rats. Am Rev Respir Dis 1985; 132: 880-884.

20. Broccard AF, Hotchkiss JR, Kuwayama N, et al. Consequences of vascular flow on lung injury induced by mechanical ventilation. Am J Respir Crit Care Med 1998; 157: 1935-1942.

21. Chiumello D, Goesev P, Slutsky AS. Mechanical ventilation affects local and systemic cytokines in an animal model of acute respiratory distress syndrome. Am J Respir Crit Care Med 1999; 160: 109-116.

22. Liu JM, De Roberstis E, Blomquist S, Dahm PL, Svantensson C, Jonson B. Elastic pressure-volume curves of the respiratory system reveal a high tendency to lung collapse in young pigs. Intensive Care Med 1999; 25: 1140-1146.

23. Jonson B, Richard JC, Straus C, Mancebo J, Lemaire F, Brochard L. Pressure-volume curves and compliance in acute lung injury: evidence of recruitment above the lower inflection point. Am J Respir Crit Care Med 1999; 159: 1172-1178.

24. Servillo G, Svantesson C, Beydon L, et al. Pressure-volume curves in acute respiratory failure: automated low flow inflation versus occlusion. Am J Respir Crit Care Med 1997; 155: $1629-1636$.

25. Jonson B, Svantesson C. Elastic pressure-volume curves: what information do they convey? Thorax 1999; 54: 82-87.

26. Vieira SR, Puybasset L, Lu Q, et al. A scanographic assessment of pulmonary morphology in acute lung injury. Significance of the lower inflection point detected on the lung pressure-volume curve. Am J Respir Crit Care Med 1999; 159: 1612-1623.

27. Lu Q, Vieira SR, Richecoeur J, et al. A simple automated method for measuring pressure-volume curves during mechanical ventilation. Am J Respir Crit Care Med 1999; 159: 275-282.

28. Vieira SR, Puybasset L, Richecoeur J, et al. A lung computed tomographic assessment of positive end-expiratory pressure-induced lung overdistension. Am J Respir Crit Care Med 1998; 158: 1571-1577.

29. Puybasset L, Cluzel P, Chao N, Slutsky AS, Coriat P, Rouby JJ. A computed tomography scan assessment of regional lung volume in acute lung injury. The CT Scan ARDS Study Group. Am J Respir Crit Care Med 1998; 158: 1644-1655.

30. Hickling KG. The pressure-volume curve is greatly modified by recruitment. A mathematical model of ARDS lungs. Am J Respir Crit Care Med 1998; 158: 194-202.

31. Mead J, Takishima T, Leith D. Stress distribuition in lungs: a model of pulmonary elasticity. J Appl Physiol 1970; 28: 596-608.

32. Mathieu-Costello OA, West JB. Are pulmonary capillaries susceptible to mechanical stress? Chest 1994; 105: 102S$107 \mathrm{~S}$.

33. Fu Z, Costello ML, Tsukimoto K, et al. High lung volume increases stress failure in pulmonary capillaries. $J$ Appl Physiol 1992; 73: 123-133.

34. West JB, Tsukimoto K, Mathieu-Costello O, Prediletto R. Stress failure in pulmonary capillaries. J Appl Physiol 1991; 70: 1731-1742.

35. Gaver DP, Samsel RW, Solway J. Effects of surface tension and viscosity on airway reopening. J Appl Physiol 1990; 69: 74-85.

36. Kamm RD, Schroter RC. Is airway closure caused by a liquid film instability? Respir Physiol 1989; 75: 141-156.

37. Gefen A, Elad D, Shiner RJ. Analysis of stress distribuition in the alveolar septa of normal and simulated emphysematic lungs. J Biomech 1999; 32: 891-897.

38. Oswari J, Matthay MA, Margulies SS. Keratinocyte growth factor reduces alveolar epithelial susceptibility to in vitro 
mechanical deformation. Am J Physiol Lung Cell Mol Physiol 2001; 281: L1068-1077.

39. Ranieri VM, Slutsky AS. Respiratory physiology and acute lung injury: the miracle of Lazarus. Intensive Care Med 1999; 25: $1040-1043$.

40. Hickling KG. Best compliance during a decremental, but not incremental, positive end-expiratory pressure trial is related to open-lung positive end-expiratory pressure: a mathematical model of acute respiratory distress syndrome lungs. Am J Respir Crit Care Med 2001; 163: 69-78.

41. Crotti S, Mascheroni D, Caironi P, et al. Recruitment and derecruitment during acute respiratory failure: a clinical study. Am J Respir Crit Care Med 2001; 164: 131-140.

42. Pelosi P, Goldner M, McKibben A, et al. Recruitment and derecruitment during acute respiratory failure: an experimental study. Am J Respir Crit Care Med 2001; 164 : 122-130.

43. Ranieri VM, Zhang H, Mascia L, et al. Pressure-time curve predicts minimally injurious ventilatory strategy in an isolated rat lung model. Anesthesiology 2000; 93: 13201328.

44. Ranieri VM, Brienza N, Santostasi S, et al. Impairment of lung and chest wall mechanics in patients with acute respiratory distress syndrome: role of abdominal distension. Am J Respir Crit Care Med 1997; 156: 1082-1091.

45. Bates JHT, Rossi A, Milic-Emili J. Analysis of the behavior of the respiratory system with constant inspiratory flow. J Appl Physiol 1985; 58: 1840-1848.

46. D'Angelo E, Robatto FM, Calderini E, et al. Pulmonary and chest wall mechanics in anesthetized paralyzed humans. J Appl Physiol 1991; 70: 2602-2610.

47. Eissa NT, Ranieri VM, Chasse M, Robatto FM, Braidy J, Milic-Emili J. Analysis of the behaviour of the respiratory system in ARDS patients: Effects of flow, volume and time. J Appl Physiol 1991; 70: 2719-2729.

48. Jonson B, Beydon L, Brauer K, Mansson C, Valind S, Grytzell H. Mechanics of respiratory system in healthy anesthetized humans with emphasis on viscoelastic properties. J Appl Physiol 1993; 75: 132-140.

49. De Perrot M, Imai Y, Volgyesi GA, et al. Effect of ventilator-induced lung injury on the development of reperfusion injury in a rat lung transplant model. $J$ Thorac Cardiovasc Surg 2002; 124: 1137-1144.

50. Nakane M, Imai Y, Kajikawa O, et al. Stress index strategy: analysis of dynamic airway opening pressure-time curve may be a useful tool to protect rabbits from VILI. Am J Respir Crit Care Med 2002; 165: A680.

51. Grasso S, Terragni P, Mascia L, et al. Dynamic airway pressure/time curve (stress index) in experimental ARDS. Intensive Care Med 2002; 28: A727.

52. Grasso S, Mascia L, Trotta $\mathrm{T}$, et al. Dynamic airway pressure/time curve analysis to realize lung protective ventilatory strategy in ARDS patients. Intensive Care Med 2000; 26: A449.

53. Grasso S, Mascia L, Capobianco S, et al. Protective ventilatory strategy: "NIH" vs "Static P-V curves" vs "Stress Index" protocol. Intensive Care Med 2000; 26: A619.

54. Neve V, de la Roque ED, Leclerc F, et al. Ventilator-induced overdistension in children: dynamic versus low-flow inflation volume-pressure curves. Am J Respir Crit Care Med 2000; 162: 139-147.

55. Gama de Abreu M, Heintz M, Heller A, Szechenyi R, Albrecht DM, Koch T. One lung ventilation with high tidal volumes and zero positive end-expiratory pressure is injurious in the isolated rabbit lung model. Anesth Analg 2003; 96: 220-228. 